Journal of Developmental

Origins of Health and Disease

www.cambridge.org/doh

\section{Characterising and monitoring preconception health in England: a review of national population-level indicators and core data sources}

\author{
Danielle A.J.M. Schoenaker ${ }^{1,2}$ (1), Judith Stephenson ${ }^{3}$, Anne Connolly ${ }^{4}$, \\ Sally Shillaker ${ }^{5}$, Sarah Fishburn ${ }^{6}$, Mary Barker ${ }^{2,7}$, Keith M. Godfrey ${ }^{2,7,8}$, \\ Nisreen A. Alwan ${ }^{1,2,9}$ and the UK Preconception Partnership
}

Stephenson J, Connolly A, Shillaker Fishburn S, Barker M, Godfrey KM, Alwan NA, and the UK Preconception Partnership. (2022) Characterising and monitoring preconception health in England: a review of national population-level indicators and core data sources. Journal of Developmental Origins of Health and Disease 13: 137-150. doi: 10.1017/ S2040174421000258

Received: 6 January 2021

Revised: 19 April 2021

Accepted: 20 April 2021

First published online: 4 June 2021

\section{Keywords:}

Preconception; surveillance; indicators; reproductive health

Address for correspondence: Danielle Schoenaker, School of Primary Care, Population Sciences and Medical Education, University of Southampton, Southampton General Hospital, Tremona Road, Southampton SO16 6YD, United Kingdom. Email: D.Schoenaker@soton.ac.uk

${ }^{1}$ School of Primary Care, Population Sciences and Medical Education, Faculty of Medicine, University of Southampton, Southampton, UK; ${ }^{2}$ NIHR Southampton Biomedical Research Centre, University of Southampton and University Hospital Southampton NHS Foundation Trust, Southampton, UK; ${ }^{3}$ Elizabeth Garrett Anderson Institute for Women's Health, University College London, London, UK; ${ }^{4}$ Bevan Healthcare, Bradford, UK; ${ }^{5}$ Academy of Research and Improvement, Solent NHS Trust, Portsmouth, UK; ${ }^{6}$ South East Clinical Delivery and Networks, NHS England and Improvement, Oxford, UK; ${ }^{7} \mathrm{MRC}$ Lifecourse Epidemiology Unit, University of Southampton, Southampton, UK; ${ }^{8}$ Human Development and Health, Faculty of Medicine, University of Southampton, Southampton, UK and ${ }^{9}$ NIHR Applied Research Collaboration Wessex, Southampton, UK

\begin{abstract}
Initiatives to optimise preconception health are emerging following growing recognition that this may improve the health and well-being of women and men of reproductive age and optimise health in their children. To inform and evaluate such initiatives, guidance is required on indicators that describe and monitor population-level preconception health. We searched relevant databases and websites (March 2021) to identify national and international preconception guidelines, recommendations and policy reports. These were reviewed to identify preconception indicators. Indicators were aligned with a measure describing the prevalence of the indicator as recorded in national population-based data sources in England. From 22 documents reviewed, we identified 66 indicators across 12 domains. Domains included wider (social/economic) determinants of health; health care; reproductive health and family planning; health behaviours; environmental exposures; cervical screening; immunisation and infections; mental health, physical health; medication and genetic risk. Sixty-five of the 66 indicators were reported in at least one national routine health data set, survey or cohort study. A measure of preconception health assessment and care was not identified in any current national data source. Perspectives from three (healthcare) professionals described how indicator assessment and monitoring may influence patient care and inform awareness campaign development. This review forms the foundation for developing a national surveillance system for preconception health in England. The identified indicators can be assessed using national data sources to determine the population's preconception needs, improve patient care, inform and evaluate new campaigns and interventions and enhance accountability from responsible agencies to improve preconception health.
\end{abstract}

(c) The Author(s), 2021. Published by Cambridge University Press in association with International Society for Developmental Origins of Health and Disease. This is an Open Access article, distributed under the terms of the Creative Commons Attribution licence (http://creativecommons.org/licenses/by/4.0/), which permits unrestricted re-use, distribution, and reproduction in any medium, provided the original work is properly cited.

\section{CAMBRIDGE} UNIVERSITY PRESS

\section{Introduction}

The health and health behaviours of women and men prior to pregnancy are key determinants of a successful pregnancy and the health of women, men and their offspring in the short- and long-term. ${ }^{1,2}$ There is now ample evidence from developmental biology and epidemiological studies that improving preconception health represents an opportunity to reduce maternal and infant mortality and morbidity, prevent non-communicable disease in parents themselves and their offspring and improve the overall health of at least two generations. The importance of optimal preconception health has been recognised in many national and international guidelines, position statements and policy reports. These provide clinical guidance on providing preconception care (PCC) to individuals planning pregnancy, ${ }^{3-9}$ outline how primary care, maternity and community services may integrate PCC into existing services ${ }^{5,6,9-12}$ and call for continued efforts to improve the health of the population more broadly. ${ }^{4-6,9,10,12,13}$

While there has to date been little progress on widespread implementation of PCC and preconception health programmes and interventions, advocacy for preconception health initiatives is increasing internationally. Examples include a focus on the health of girls and women during the adolescent and reproductive years in the United Nations 2030 Agenda for Sustainable Development $^{14}$ and in the Global Strategy for Women's, Children's and Adolescent's 
Health $2016-2030 .{ }^{15}$ As a result, interventions that increase awareness of the importance of preconception health and that promote pregnancy planning and preparation are likely to be developed to meet the goals set out in these global strategies. National and local programmes and interventions are emerging ${ }^{10,16,17}$ and already being tested and implemented in some countries such as the USA, China, Belgium and the Netherlands. ${ }^{18-20}$ To inform and evaluate such initiatives and to track progress made towards optimising preconception health, there is a need for guidance on the assessment and monitoring of population-level indicators that measure the state of preconception health.

In the UK, the Preconception Partnership was established in 2018 as a multi-disciplinary advocacy coalition with the aim to improve preconception health. The Partnership has set out a conceptual framework for reporting and monitoring of preconception indicators in England based on a set of routinely collected data sets, or 'core metrics'. ${ }^{21}$ We define preconception indicators as medical, behavioural and social risk factors or exposures as well as wider determinants of health that may impact potential future pregnancies among all women and men of reproductive age. ${ }^{10,17}$ National data on these indicators would facilitate characterisation of the nation's preconception health, monitoring of trends and identification of inequalities, to incite action from local and national governments and organisations to deliver resources for effective and appropriate interventions. Internationally, there is currently no comprehensive and recognised set of preconception indicators that could fulfil these functions using data sources available at a national level in countries such as England.

To inform the reporting of population-level preconception health in England, we aimed to conduct a review of relevant guideline and policy documents to identify a comprehensive set of indicators and to map these against national data sources currently available. Moreover, we provide perspectives from (healthcare) professionals to illustrate how findings from population-level surveillance of preconception health may be used to inform the development of interventions and campaigns and improve patient care in primary care and community settings.

\section{Methods}

\section{Review of preconception guidelines, recommendations and} policy reports

\section{Search strategy}

An electronic search was carried out in Google and Google Scholar in March 2021 by DS, and relevant guideline repositories and websites of professional organisations/associations and ministries of health were searched, to identify publicly available preconception guidelines, recommendations, position statements and policy reports in England and internationally. The following search terms were used: Preconception; Preconceptual; Pre-pregnancy; Before pregnancy; Pregnancy planning; Preparing for pregnancy AND Guidelines; Recommendations; Policy.

\section{Eligibility criteria}

Guidelines, statements and policy documents aimed at the public or patients, healthcare professionals and governments reporting on preconception indicators that could be assessed in individuals (i.e. not for example at health service or household level) were considered relevant. In addition, preconception recommendations published in English by other countries were included to identify additional preconception indicators reported internationally but not included in documents relevant to England. International documents were not included if no additional preconception indicators were identified beyond those already reported in documents relevant to England. Electronically available information considered relevant for identifying preconception indicators included documents and other online information sources from government organisations, national healthcare organisations, professional bodies and societies and charitable organisations.

\section{Data extraction}

Key features of the guidelines, statements and policy documentation (including the overall topic, the target audience(s) and country) and all preconception indicators mentioned in these sources were extracted by DS. The identified preconception indicators were grouped into overarching domains based on shared characteristics. Measures that describe the prevalence of the indicators were formulated based on the guidelines and recommendations reviewed.

\section{Aligning preconception indicators with core data sources}

Following identification of preconception indicators, populationbased national data sources from which prevalence data on indicators could be extracted were identified through known UK websites providing information on national data sets, national data reports or surveillance of health indicators. Examples include websites from NHS Digital, ${ }^{22}$ the UK Data Service, ${ }^{23}$ Public Health Outcomes Framework ${ }^{24}$ and Office for National Statistics. ${ }^{25}$ Eligible data sources included national routine data sets, national surveys and national cohort studies. We excluded data sources if they did not include individual-level data for women and/or men of reproductive age (defined as aged 16-45 years ${ }^{3}$ ), or if data were not directly available or accessible through online data reports or tools. For each data source, information was summarised on the representativeness of the population, the method and frequency of data collection, reporting of relevant preconception indicators and data access.

Preconception indicators were aligned with a data source where possible. If an indicator was not available but a comparable measure was reported in a data source, the comparable measure was included to reflect an approximation of the preconception indicator as specified in guideline and policy documents.

\section{Results}

\section{Preconception indicators}

The search for publicly available guidance on preconception health in England identified 15 clinical guidance or recommendation documents, three position statements, two policy reports, one eLearning programme and online information from one charitable organisation (Supplementary Table S1). Guidance was aimed at healthcare professionals, commissioners and providers or the public and was relevant to the general population of reproductive-aged women and men, individuals planning pregnancy and/or populations with pre-existing medical conditions or at high risk of an adverse pregnancy or birth outcome. International guidance related to preconception health that reported additional indicators not described in documents relating to England included clinical preconception care recommendations from the USA and Canada, a position statement from Australia and a policy brief from the World Health Organization (WHO) (Supplementary Table S1). Examples of international documents that were 
identified but that did not report preconception indicators not already reported in documents relevant to England included the Federation of Obstetric and Gynaecological Societies of India (FOGSI) Good Clinical Practice Recommendation on Preconception Care, ${ }^{26}$ a call to action for preconception health promotion and care by the Ontario Public Health Association ${ }^{27}$ and a report commissioned by NHS Greater Glasgow and Clyde on Preconception health, education and care in Scotland. ${ }^{28}$

Preconception indicators were grouped into 12 domains containing a total of 66 indicators (Table 1). Detailed measures formulated based on guidelines and recommendations can be found in Supplementary Table S2. Several preconception indicators could be assessed using multiple measures, and separate measures were formulated for women and men, for high-risk groups and categorical indicators (such as body mass index classifications) based on clinical guidance. While guidance was mainly focused on preconception health of women, some clinical guidelines, ${ }^{3,4,6,12,29,30}$ a Clinical Effectiveness Unit position statement ${ }^{12}$ and Tommy's charity ${ }^{31}$ reported on preconception indicators relevant to men.

\section{Core data sources}

National data sources with relevant information on preconception health included 13 routine health data sets, five surveys and two cohort studies (Table 2). Routine health data sets cover data reported in primary care, hospital, maternity, community and specialist health services, disease registries and the census. National surveys are repeated cross-sectional surveys among a random representative sample, and national cohort studies include longitudinal population-based studies following the lives of people born in a specific week or year from birth onwards at 2-10 year intervals. Data for surveys and cohort studies are collected through questionnaires, face-to-face interviews, physical examinations and biological samples. Anonymised annual data for individuals across their reproductive years are available to policymakers and researchers for the majority of data sources following protocol approval through NHS Digital or the UK Data Service (Table 2). Key statistics from most routine data sets and surveys are published online at least annually. These reports do not cover all indicators measured in each data source and are generally not aggregated specifically for women and men of reproductive age.

From the total number of 66 identified preconception indicators, 65 were reported in at least one data source (Table 1, Supplementary Table S1). Collectively, national routine data sets include data for all domains and nearly all indicators except measures on adverse childhood experiences, preconception assessment and care, pregnancy intention, dietary intake and physical activity. Data on indicators related to health behaviours including diet and physical activity are collected in detail and through validated methods in surveys and cohort studies. A measure of preconception health assessment and care was not identified in any current national data source.

\section{Discussion}

This review is the first critical step in the process of developing a national surveillance system for preconception health in England. Based on national and international guidelines and policy documents, we identified a comprehensive set of preconception indicators that can be used to characterise and monitor the health, health behaviours and their wider determinants among women and men of reproductive age using available national population-based data sources. A national picture of preconception health can improve our understanding of the population's preconception needs, inform the development and evaluation of new campaigns and interventions, and thereby facilitate translation of evidence into actions to improve preconception health.

Existing public health surveillance systems in England, such as those managed by Public Health England ${ }^{24}$ and the Office for National Statistics, ${ }^{25}$ produce and publish regularly updated population-level data on a large number of indicators across a broad range of topics. These include wider determinants of health and indicators relevant to preconception health such as obesity and smoking prevalence. We present an extension to these existing surveillance efforts based on indicators that are relevant to people of reproductive age and that are recommended as indicators of optimal preconception health and care based on clinical guidance and policy reports. Our review identified a set of 66 preconception indicators across 12 domains covering wider determinants of health and medical, behavioural, social and environmental risk factors. Measures that describe the prevalence of each indicator were formulated based on guidelines and recommendations reviewed and are specific to women or men and high-risk groups where relevant.

Although not all indicators identified in our review are currently recorded in a data source representative of the English population of women and men of reproductive age, 65 of the 66 indicators are assessed and can already be described and monitored at a national level. A measure of preconception health assessment and care is not currently captured in any national data source, and pregnancy intention is not recorded in a routine data set or annual survey. These measures should be considered for inclusion in routine data sets or annual surveys in the future to allow for comprehensive and timely monitoring and evaluation.

For the first time, we describe indicators relevant to men's preconception health and indicators that can be measured at a national level rather than a local or state level. Indicators were aligned with a range of national data sources, including routine data sets, surveys and cohort studies. The majority of indicators were recorded in at least one routine health data set commissioned by the National Health Service (NHS), which is a governmentsponsored universal healthcare system and provider of the majority of care in England. The advantage of monitoring preconception indicators through a combination of these data sources from primary care, hospital, maternity, community and specialist health services is the wide population coverage with information on nearly all residents of England. National surveys and cohort studies complement routine data with indicators on health behaviours such as diet and physical activity comprehensively assessed among large nationally representative samples of the population.

Population-level surveillance indicators for preconception health and care have also been identified in the US by the National Preconception Health and Health Care (PCHHC) Initiative. ${ }^{32}$ The indicator selection process by the PCHHC Initiative involved a consensus-based selection of 11 broad priority areas (domains), a review of state-based data systems and identification of available measures relevant to each domain. Compared with the domains defined in our review, the PCHHC Initiative did not select environmental exposures, cervical screening, medication and genetic risk as priority areas, but identified self-rated general health status as an additional priority area and separated the health behaviours domain into tobacco, alcohol and substance use and nutrition and physical activity. A total of 96 measures were identified by the PCHHC Initiative, and these were reduced to 45 
Table 1. Summary overview of identified preconception indicators and their recording in national data sources

\begin{tabular}{|c|c|c|c|c|c|c|c|c|c|}
\hline \multirow[b]{2}{*}{ Domain } & \multirow[b]{2}{*}{ Indicator } & \multicolumn{4}{|c|}{ INDICATOR SOURCE (REFERENCE) } & \multirow[b]{2}{*}{ E-Learning } & \multicolumn{3}{|c|}{ POSSIBLE DATA SOURCE } \\
\hline & & $\begin{array}{l}\text { Clinical } \\
\text { guidance }\end{array}$ & $\begin{array}{l}\text { Policy } \\
\text { report }\end{array}$ & $\begin{array}{l}\text { Position } \\
\text { statement }\end{array}$ & $\begin{array}{l}\text { Charitable } \\
\text { organisation }\end{array}$ & & $\begin{array}{l}\text { National routine data } \\
\text { set }\end{array}$ & National survey & $\begin{array}{l}\text { National } \\
\text { cohort study }\end{array}$ \\
\hline \multirow[t]{9}{*}{$\begin{array}{l}\text { Wider determinants of } \\
\text { health }\end{array}$} & Ethnicity & & 10 & & & & $\begin{array}{l}\text { RCGP, CPRD, MSDS, } \\
\text { HES, ONS, NDA }\end{array}$ & $\begin{array}{l}\text { HSE, NDNS, } \\
\text { ONS, ALAS, }\end{array}$ & MCS, BCS70 \\
\hline & Education & & 10 & & & & HES, ONS & $\begin{array}{l}\text { HSE, ONS, } \\
\text { ALAS, }\end{array}$ & MCS, BCS70 \\
\hline & Employment & & 10 & & & & $\begin{array}{l}\text { RCGP, CPRD, MSDS, } \\
\text { HES, ONS }\end{array}$ & HSE, ALAS, ONS & MCS, BCS70 \\
\hline & Financial security & & 10 & & & & ONS & ONS & \\
\hline & Housing & & 10 & & & & ONS & ONS & BCS70 \\
\hline & Deprivation & & 10 & & & & $\begin{array}{l}\text { RCGP, CPRD, MSDS, } \\
\text { HES, ONS }\end{array}$ & NDNS, ONS & MCS, BCS70 \\
\hline & Complex social factors & & 10 & & & & MSDS & & \\
\hline & Adverse childhood experiences & & 10 & & & & & ONS & \\
\hline & Migrant health factors & & 10 & & & & RCGP, CPRD ONS & ONS & \\
\hline \multirow[t]{3}{*}{ Health care } & Preconception assessment and care & $3,6,42,43$ & & & & & & & \\
\hline & $\begin{array}{l}\text { Routine general practitioner (GP) } \\
\text { check-up }\end{array}$ & 3 & & & & & RCGP, CPRD & & \\
\hline & Routine dental appointment & 7 & & & & 44 & NHSDS & & \\
\hline \multirow[t]{2}{*}{$\begin{array}{l}\text { Emotional and social } \\
\text { health and support }\end{array}$} & $\begin{array}{l}\text { Domestic abuse (physical and emo- } \\
\text { tional) }\end{array}$ & 5 & 10 & & 31 & 44 & RCGP, CPRD & ONS & MCS, BCS70 \\
\hline & Support network/social support & & 10 & & 31 & & MSDS & & \\
\hline \multirow{9}{*}{$\begin{array}{l}\text { Reproductive health and } \\
\text { family planning }\end{array}$} & Pregnancy intention & $3,4,30$ & & & 31 & & & Natsal & \\
\hline & Maternal age & 3,30 & 10 & & & & $\begin{array}{l}\text { RCGP, CPRD, MSDS, } \\
\text { HES }\end{array}$ & & MCS, BCS70 \\
\hline & Paternal age & 29 & & & & & Birth registrations & & \\
\hline & Interpregnancy interval & 3 & & & & & $\begin{array}{l}\text { RCGP, CPRD, MSDS, } \\
\text { HES }\end{array}$ & & \\
\hline & Obstetric history & $\begin{array}{l}3,4,7,8 \\
42,43\end{array}$ & 10 & & 31 & & $\begin{array}{l}\text { RCGP, CPRD, HES, } \\
\text { MSDS, NCARDRS }\end{array}$ & Natsal & MCS, BCS70 \\
\hline & Previous breastfeeding experiences & & & & & 44 & CSDS & & BCS70 \\
\hline & Fertility problems & 3,30 & & & & 44 & RCGP, CPRD, HFEA & & \\
\hline & Contraception & $\begin{array}{l}3,4,42 \\
45,46\end{array}$ & 10 & 12 & 31 & 44 & RCGP, CPRD, SRHAD & HSE & MCS \\
\hline & Assisted reproductive technology & 3,30 & & 12 & & & HFEA & & \\
\hline
\end{tabular}


Table 1. (Continued)

\begin{tabular}{|c|c|c|c|c|c|c|c|c|c|}
\hline \multirow[b]{2}{*}{ Domain } & \multirow[b]{2}{*}{ Indicator } & \multicolumn{4}{|c|}{ INDICATOR SOURCE (REFERENCE) } & \multirow[b]{2}{*}{ E-Learning } & \multicolumn{3}{|c|}{ POSSIBLE DATA SOURCE } \\
\hline & & $\begin{array}{l}\text { Clinical } \\
\text { guidance }\end{array}$ & $\begin{array}{l}\text { Policy } \\
\text { report }\end{array}$ & $\begin{array}{l}\text { Position } \\
\text { statement }\end{array}$ & $\begin{array}{l}\text { Charitable } \\
\text { organisation }\end{array}$ & & $\begin{array}{l}\text { National routine data } \\
\text { set }\end{array}$ & National survey & $\begin{array}{l}\text { National } \\
\text { cohort study }\end{array}$ \\
\hline \multirow[t]{11}{*}{ Health behaviours } & Folic acid supplementation & $3-5,30,42$ & 10 & 12 & 31 & 44 & MSDS, NDA & & \\
\hline & Other vitamin supplementation & 4,5 & & 11 & 31 & 44 & & NDNS & \\
\hline & Vitamin deficiency & $4,5,7$ & 9 & 13 & 31 & & RCGP, CPRD & NDNS & \\
\hline & Dietary intake & $\begin{array}{l}3-5,30 \\
47,48\end{array}$ & 10 & 12 & 31 & 44 & & NDSD & BCS70 \\
\hline & Physical activity & 4,48 & 10 & & 31 & 44 & & ALAS & \\
\hline & Weight & $3-5,30,48$ & 10 & 12 & 31 & 44 & RCGP, CPRD & $\begin{array}{l}\text { Natsal, HSE, } \\
\text { NDNS, ALAS, }\end{array}$ & MCS, BCS70 \\
\hline & Eating disorder & 45 & & & 31 & & RCGP, CPRD & & \\
\hline & Tobacco use & $3-5,30$ & 10 & 12 & 31 & 44 & RCGP, CPRD & $\begin{array}{l}\text { Natsal, HSE, } \\
\text { ONS }\end{array}$ & MCS, BCS70 \\
\hline & Second-hand smoke exposure & 4,30 & & & 31 & & & HSE & \\
\hline & Alcohol consumption & $3-5,30$ & 10 & 12 & 31 & 44 & RCGP, CPRD & $\begin{array}{l}\text { Natsal, HSE, } \\
\text { ONS }\end{array}$ & MCS, BCS70 \\
\hline & Substance use & $3-5,30$ & 10 & 12 & 31 & 44 & RCGP, CPRD & Natsal & MCS \\
\hline Environmental exposures & $\begin{array}{l}\text { Toxic or hazardous substances or } \\
\text { exposures }\end{array}$ & $3,30,49$ & & & & & $\begin{array}{l}\text { RCGP, CPRD, MSDS, } \\
\text { HES, ONS }\end{array}$ & ONS, NDNS & MCS, BCS70 \\
\hline Cervical screening & Cervical screening & 3,30 & 10 & 12 & 31 & 44 & RCGP, CPRD & & \\
\hline \multirow{2}{*}{$\begin{array}{l}\text { Immunisation and infec- } \\
\text { tions }\end{array}$} & Immunisation & $3,4,8,30$ & 10 & 12 & 31 & 44 & RCGP, CPRD & & \\
\hline & Sexually transmitted diseases & 3,30 & 10 & 12 & 31 & 44 & RCGP, CPRD & Natsal & \\
\hline \multirow[t]{3}{*}{ Mental health conditions } & Mental health problem & $3-5,29$ & 10 & 12 & 31 & 44 & RCGP, CPRD, HES, ONS & $\begin{array}{l}\text { Natsal, HSE, } \\
\text { ONS }\end{array}$ & MCS, BCS70 \\
\hline & $\begin{array}{l}\text { Previous antenatal or postnatal men- } \\
\text { tal illness }\end{array}$ & 7 & & & & 44 & RCGP, CPRD, CSDS & & \\
\hline & Severe mental health conditions & 3 & & & & & RCGP, CPRD, HES & & \\
\hline \multirow{10}{*}{$\begin{array}{l}\text { Physical health condi- } \\
\text { tions }\end{array}$} & Epilepsy & 3,4 & & & 31 & 44 & RCGP, CPRD, HES & Natsal & BCS70 \\
\hline & Diabetes mellitus & $3,4,29,42$ & & 12 & 31 & 44 & RCGP, CPRD, HES, NDA & Natsal, HSE & BCS70 \\
\hline & Polycystic ovary syndrome & & & & 31 & & RCGP, CPRD, HES & Natsal & \\
\hline & Endometriosis & 30,50 & & & 31 & & RCGP, CPRD, HES & & \\
\hline & Thyroid disease & 3,30 & & & & & RCGP, CPRD, HES & Natsal & \\
\hline & Chronic hypertension & 3,4 & & 12 & & 44 & RCGP, CPRD, HES & Natsal, HSE & BCS70 \\
\hline & Cardiovascular disease & $3,4,46$ & & & & & RCGP, CPRD, HES & Natsal & \\
\hline & Previous thromboembolism & 3 & & & & & RCGP, CPRD, HES & & \\
\hline & Renal disease & 3 & & & & & RCGP, CPRD, HES & & \\
\hline & Asthma & 3,4 & & & 31 & & RCGP, CPRD, HES & & BCS70 \\
\hline
\end{tabular}


Table 1. (Continued)

\begin{tabular}{|c|c|c|c|c|c|c|c|c|c|}
\hline \multirow[b]{2}{*}{ Domain } & \multirow[b]{2}{*}{ Indicator } & \multicolumn{4}{|c|}{ INDICATOR SOURCE (REFERENCE) } & \multirow[b]{2}{*}{ E-Learning } & \multicolumn{3}{|c|}{ 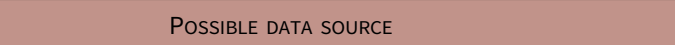 } \\
\hline & & $\begin{array}{l}\text { Clinical } \\
\text { guidance }\end{array}$ & $\begin{array}{l}\text { Policy } \\
\text { report }\end{array}$ & $\begin{array}{l}\text { Position } \\
\text { statement }\end{array}$ & $\begin{array}{l}\text { Charitable } \\
\text { organisation }\end{array}$ & & $\begin{array}{l}\text { National routine data } \\
\text { set }\end{array}$ & National survey & $\begin{array}{l}\text { National } \\
\text { cohort study }\end{array}$ \\
\hline & Rheumatological conditions & 3 & & & & 44 & RCGP, CPRD, HES & & \\
\hline & Inflammatory bowel disease & 3,51 & & & & & RCGP, CPRD, HES & & \\
\hline & Sickle-cell disease or thalassaemia & 3,4 & & 12 & & & $\begin{array}{l}\text { RCGP, CPRD, HES, } \\
\text { NCARDRS }\end{array}$ & & \\
\hline & Hepatitis B & 3,30 & & & & & RCGP, CPRD & & \\
\hline & Lupus & 8 & & & & & RCGP, CPRD, HES & & \\
\hline & Phenylketonuria (PKU) & 6 & & & & & $\begin{array}{l}\text { RCGP, CPRD, HES, } \\
\text { NCARDRS }\end{array}$ & & \\
\hline & Developmental disability & 8 & & & & & $\begin{array}{l}\text { RCGP, CPRD, HES, } \\
\text { NCARDRS }\end{array}$ & & \\
\hline & Cancer & 30 & & & & & $\begin{array}{l}\text { RCGP, CPRD, HES, } \\
\text { NCARDRS, NCSRAS }\end{array}$ & & BCS70 \\
\hline & Female genital mutilation & 3,30 & & & & & $\begin{array}{l}\text { RCGP, CPRD, MSDS, } \\
\text { HES }\end{array}$ & & \\
\hline & Pelvic floor dysfunction & 52 & & & & 44 & RCGP, CPRD, HES & & \\
\hline \multirow[t]{2}{*}{ Medication } & Overall use & 3,30 & & & 31 & & RCGP, CPRD, HES & HSE & \\
\hline & $\begin{array}{l}\text { Not recommended when planning } \\
\text { pregnancy }\end{array}$ & $3-5$ & & 12 & & & RCGP, CPRD, HES & & \\
\hline \multirow[t]{3}{*}{ Genetic risk } & $\begin{array}{l}\text { Personal or family history of a generic } \\
\text { disorder }\end{array}$ & $3,4,30$ & 10 & 12 & 31 & & RCGP, CPRD, NCARDRS & & \\
\hline & $\begin{array}{l}\text { Previous pregnancy affected by an } \\
\text { inherited genetic disorder }\end{array}$ & 3 & & & & & RCGP, CPRD, NCARDRS & & \\
\hline & Family history of diabetes & 29,42 & & & & & RCGP, CPRD & & \\
\hline
\end{tabular}

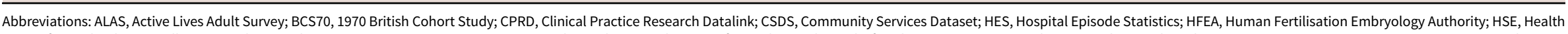

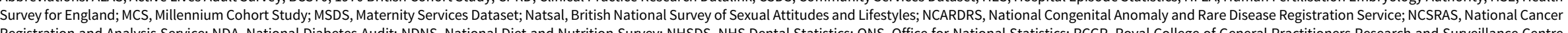
e gistration and Analysis Senice, NDA, National Diabetes Audi, NDN, 
Table 2. Core data sources for the reporting and monitoring of preconception indicators in England

\begin{tabular}{|c|c|c|c|}
\hline Data source & Description & Data access & More information \\
\hline \multicolumn{4}{|c|}{ National routine data sets } \\
\hline $\begin{array}{l}\text { Royal College of } \\
\text { General Practitioners } \\
\text { (RCGP) Research and } \\
\text { Surveillance Centre } \\
\text { (RSC) database }\end{array}$ & $\begin{array}{l}\text { The RCGP RSC is a network of primary } \\
\text { care practices, which extracts data from } \\
\text { the computerised medical record systems } \\
\text { of over } 1,700 \text { practices in England. Up to } \\
\text { early } 2020 \text {, data covered approximately } \\
5 \% \text { of the population in England, }{ }^{53} \text { which } \\
\text { has increased substantially as the RCGP } \\
\text { RSC expands. Includes individual-level } \\
\text { annual data of women and men aged } \\
16-45 \text { years who are registered with a } \\
\text { general practitioner (GP) practice partici- } \\
\text { pating in the RCGP RSC network on wider } \\
\text { determinants of health, health care, repro- } \\
\text { ductive health and family planning, health } \\
\text { behaviours, cervical screening, infections, } \\
\text { mental and physical health conditions, } \\
\text { medication and genetic risk. }\end{array}$ & $\begin{array}{l}\text { Researchers may access anonymised indi- } \\
\text { vidual-level data following protocol } \\
\text { approval. }\end{array}$ & $\begin{array}{l}\text { https://www.rcgp.org.uk/clinical-and- } \\
\text { research/our-programmes/research- } \\
\text { and-surveillance-centre.aspx } \\
\text { [accessed 22/03/2021] }\end{array}$ \\
\hline $\begin{array}{l}\text { Clinical Practice } \\
\text { Research Datalink } \\
\text { (CPRD) }\end{array}$ & $\begin{array}{l}\text { Research service by the Department of } \\
\text { Health and Social Care. Collects data from } \\
\text { the computerised medical record systems of } \\
\text { a network of GP practices in the UK. Data } \\
\text { cover approximately } 7 \% \text { of the UK popula- } \\
\text { tion. }{ }^{54} \text { Includes individual-level annual data } \\
\text { of women and men aged } 16-45 \text { years who } \\
\text { are registered with a GP practice participat- } \\
\text { ing in the CPRD network on wider determi- } \\
\text { nants of health, health care, reproductive } \\
\text { health and family planning, health behav- } \\
\text { iours, cervical screening, infections, mental } \\
\text { and physical health conditions, medication } \\
\text { and genetic risk. }\end{array}$ & $\begin{array}{l}\text { Researchers may access anonymised } \\
\text { individual-level data following protocol } \\
\text { approval. }\end{array}$ & $\begin{array}{l}\text { https://www.cprd.com/ } \\
\text { [accessed 22/03/2021] }\end{array}$ \\
\hline $\begin{array}{l}\text { Maternity Services } \\
\text { Dataset (MSDS) }\end{array}$ & $\begin{array}{l}\text { Mandatory data collection by the National } \\
\text { Health Service (NHS) for all providers of } \\
\text { NHS Maternity Services in England. Data } \\
\text { are collected from the point of the first } \\
\text { booking appointment until mother and } \\
\text { baby(s) are discharged from maternity } \\
\text { services. Includes individual-level annual } \\
\text { data of pregnant women aged 16-45 years } \\
\text { on wider determinants of health, emo- } \\
\text { tional and social health and support, } \\
\text { reproductive health and family planning, } \\
\text { health behaviours, infections, mental and } \\
\text { physical health conditions and genetic risk. }\end{array}$ & $\begin{array}{l}\text { Access to anonymised individual-level } \\
\text { data is available to researchers following } \\
\text { protocol approval through the NHS Digital } \\
\text { Data Access Request Service, and key } \\
\text { statistics are published online at least } \\
\text { annually. }\end{array}$ & $\begin{array}{l}\text { https://digital.nhs.uk/data-and- } \\
\text { information/data-collections-and-data- } \\
\text { sets/data-sets/maternity-services-data- } \\
\text { set } \\
\text { [accessed 22/03/2021] }\end{array}$ \\
\hline Birth registrations & $\begin{array}{l}\text { Birth statistics are derived from informa- } \\
\text { tion recorded when live births and } \\
\text { stillbirths are registered as part of civil } \\
\text { registration, which is a legal requirement. } \\
\text { Registration of births is a service carried } \\
\text { out by the Local Registration Service in } \\
\text { partnership with the General Register } \\
\text { Office. Birth statistics for England and } \\
\text { Wales are produced by the Office for } \\
\text { National Statistics. Birth statistics include } \\
\text { individual-level annual data on the child } \\
\text { and both parents, including the country } \\
\text { of birth and age of the mother and father } \\
\text { (or second parent). }\end{array}$ & $\begin{array}{l}\text { Data are held by the Office for National } \\
\text { Statistics, and key statistics are published } \\
\text { online annually. }\end{array}$ & $\begin{array}{l}\text { https://www.ons.gov.uk/ } \\
\text { peoplepopulationandcommunity/ } \\
\text { birthsdeathsandmarriages/livebirths/ } \\
\text { datasets/birthsbyparentscharacteristics } \\
\text { [accessed 22/03/2021] }\end{array}$ \\
\hline $\begin{array}{l}\text { Hospital Episode } \\
\text { Statistics (HES) }\end{array}$ & $\begin{array}{l}\text { Data collection by the NHS containing } \\
\text { details of all admissions, outpatient } \\
\text { appointments and accident and emergency } \\
\text { attendances at NHS hospitals in England. } \\
\text { Includes individual-level annual data of } \\
\text { women and men aged } 16-45 \text { years on } \\
\text { wider determinants of health, mental and } \\
\text { physical health conditions, reproductive } \\
\text { health and medication. }\end{array}$ & $\begin{array}{l}\text { Access to anonymised individual-level } \\
\text { data is available to researchers following } \\
\text { protocol approval through the NHS Digital } \\
\text { Data Access Request Service, and key } \\
\text { statistics are published online at least } \\
\text { annually. }\end{array}$ & $\begin{array}{l}\text { https://digital.nhs.uk/data-and- } \\
\text { information/data-tools-and-services/ } \\
\text { data-services/hospital-episode-statistics } \\
\text { [accessed 22/03/2021] }\end{array}$ \\
\hline
\end{tabular}


Table 2. (Continued)

\begin{tabular}{|c|c|c|c|}
\hline Data source & Description & Data access & More information \\
\hline $\begin{array}{l}\text { Community Services } \\
\text { Dataset (CSDS) }\end{array}$ & $\begin{array}{l}\text { Mandatory collection of data by the NHS } \\
\text { for all providers of publicly funded com- } \\
\text { munity services in England, including NHS } \\
\text { Trusts, health and community centres, } \\
\text { schools, mental health trusts and local } \\
\text { authorities. Includes individual-level } \\
\text { annual data of women aged } 16-45 \text { years } \\
\text { with a previous live birth on postpartum } \\
\text { depression. }\end{array}$ & $\begin{array}{l}\text { Access to anonymised individual-level } \\
\text { data is available to researchers following } \\
\text { protocol approval through the NHS Digital } \\
\text { Data Access Request Service, and key } \\
\text { statistics are published online at least } \\
\text { annually. }\end{array}$ & $\begin{array}{l}\text { https://digital.nhs.uk/data-and- } \\
\text { information/data-collections-and-data- } \\
\text { sets/data-sets/community-services- } \\
\text { data-set } \\
\text { [accessed 22/03/2021] }\end{array}$ \\
\hline $\begin{array}{l}\text { NHS Dental Statistics } \\
\text { (NHSDS) }\end{array}$ & $\begin{array}{l}\text { Collection of data by the NHS for all NHS } \\
\text { dentists. Includes individual-level annual } \\
\text { data of women and men aged 16-45 years } \\
\text { using NHS Dental Services. }\end{array}$ & $\begin{array}{l}\text { Researchers may contact NHS Digital to } \\
\text { discuss access to anonymised individual- } \\
\text { level data, and key statistics are published } \\
\text { online annually. }\end{array}$ & $\begin{array}{l}\text { https://digital.nhs.uk/data-and- } \\
\text { information/publications/statistical/ } \\
\text { nhs-dental-statistics } \\
\text { [accessed 22/03/2021] }\end{array}$ \\
\hline $\begin{array}{l}\text { National Diabetes Audit } \\
\text { (NDA) }\end{array}$ & $\begin{array}{l}\text { Mandatory collection of data by the NHS } \\
\text { for all GP practices and specialist services } \\
\text { such as diabetes clinics, linked with HES } \\
\text { data. Includes individual-level annual data } \\
\text { of women and men aged } 16-45 \text { years on } \\
\text { wider determinants of health and diabetes } \\
\text { (including pre-existing diabetes in preg- } \\
\text { nancy). }\end{array}$ & $\begin{array}{l}\text { Researchers may contact NHS Digital to } \\
\text { discuss access to anonymised individual- } \\
\text { level data, and key statistics are published } \\
\text { online at least annually. }\end{array}$ & $\begin{array}{l}\text { https://digital.nhs.uk/data-and- } \\
\text { information/clinical-audits-and- } \\
\text { registries/national-diabetes-audit } \\
\text { [accessed 22/03/2021] }\end{array}$ \\
\hline $\begin{array}{l}\text { National Cancer } \\
\text { Registration and } \\
\text { Analysis Service } \\
\text { (NCRAS) }\end{array}$ & $\begin{array}{l}\text { Data collection services by Public Health } \\
\text { England on all cases of cancer diagnosed } \\
\text { and treated in the NHS in England based } \\
\text { on multiple data sources including records } \\
\text { from hospitals, general practices, pathol- } \\
\text { ogy laboratories and community prescrib- } \\
\text { ing services. Includes individual-level } \\
\text { annual data of women and men aged 16- } \\
45 \text { years with (prior) cancer (treatment). }\end{array}$ & $\begin{array}{l}\text { Access to anonymised individual-level } \\
\text { data is available to researchers following } \\
\text { protocol approval through the Public } \\
\text { Health England Office for Data Release } \\
\text { (ODR), and key statistics are published } \\
\text { online annually. }\end{array}$ & $\begin{array}{l}\text { https://www.cancerdata.nhs.uk } \\
\text { [accessed 22/03/2021] }\end{array}$ \\
\hline $\begin{array}{l}\text { National Congenital } \\
\text { Anomaly and Rare } \\
\text { Disease Registration } \\
\text { Service (NCARDRS) }\end{array}$ & $\begin{array}{l}\text { Data collection services by Public Health } \\
\text { England collecting data on over } 1,000 \text { dif- } \\
\text { ferent congenital anomalies and rare dis- } \\
\text { eases, such as congenital anomalies, } \\
\text { developmental disability, perinatal and } \\
\text { infant mortality. Includes individual-level } \\
\text { annual data of women and men aged 16- } \\
45 \text { years with congenital anomalies and } \\
\text { rare diseases. }\end{array}$ & $\begin{array}{l}\text { Access to anonymised individual-level } \\
\text { data is available to researchers following } \\
\text { protocol approval through the Public } \\
\text { Health England Office for Data Release } \\
\text { (ODR), and key statistics are published } \\
\text { online annually. }\end{array}$ & $\begin{array}{l}\text { https://www.gov.uk/guidance/the- } \\
\text { national-congenital-anomaly-and-rare- } \\
\text { disease-registration-service-ncardrs } \\
\text { [accessed 22/03/2021] }\end{array}$ \\
\hline $\begin{array}{l}\text { Sexual and } \\
\text { Reproductive Health } \\
\text { Activity Dataset (SRHAD) }\end{array}$ & $\begin{array}{l}\text { Data on contraception are collected on } \\
\text { behalf of Public Health England. Data col- } \\
\text { lection is mandatory for all providers of } \\
\text { NHS Sexual and Reproductive Health ser- } \\
\text { vices. Includes individual-level annual } \\
\text { data of women and men aged } 16-45 \text { years } \\
\text { who use Sexual and Reproductive Health } \\
\text { services for contraceptive reasons on } \\
\text { uptake of long-acting reversible contra- } \\
\text { ceptives, contraceptive pill and emergency } \\
\text { contraception. }\end{array}$ & $\begin{array}{l}\text { Researchers may contact NHS Digital to } \\
\text { discuss access to anonymised individual- } \\
\text { level data, and key statistics are published } \\
\text { online annually. }\end{array}$ & $\begin{array}{l}\text { https://digital.nhs.uk/data-and- } \\
\text { information/data-collections-and-data- } \\
\text { sets/data-collections/sexual-and- } \\
\text { reproductive-health-activity-data-set- } \\
\text { srhad-collection } \\
\text { [accessed 22/03/2021] }\end{array}$ \\
\hline $\begin{array}{l}\text { Human Fertilisation and } \\
\text { Embryology Authority } \\
\text { (HFEA) }\end{array}$ & $\begin{array}{l}\text { Data on fertility treatment cycles are col- } \\
\text { lected by the Human Fertilisation and } \\
\text { Embryology Authority. Data collection on } \\
\text { every fertility treatment cycle and babies } \\
\text { born as a result is mandatory for all clin- } \\
\text { ics in the UK. Includes individual-level } \\
\text { annual data of women aged } 16-45 \text { on fer- } \\
\text { tility treatments. }\end{array}$ & $\begin{array}{l}\text { Access to anonymised individual-level } \\
\text { data is freely available through the } \\
\text { Authority's website. Researchers requiring } \\
\text { linkage of data with other datasets can } \\
\text { contact the HFEA for information about } \\
\text { the process and legal requirement. }\end{array}$ & $\begin{array}{l}\text { https://www.hfea.gov.uk/about-us/our- } \\
\text { data/ } \\
\text { [accessed 18/05/2021] }\end{array}$ \\
\hline Census & $\begin{array}{l}\text { The census is a survey conducted every } 10 \\
\text { years by the Office for National Statistics } \\
\text { (ONS). Data are collected from every } \\
\text { household in England and Wales. Includes } \\
\text { individual-level data of women and men } \\
\text { aged } 16-45 \text { years on wider determinants } \\
\text { of health, and mental and physical health. }\end{array}$ & $\begin{array}{l}\text { Researchers can access the majority of } \\
\text { data through the UK Data Service, and } \\
\text { key statistics are published online. }\end{array}$ & $\begin{array}{l}\text { https://www.ons.gov.uk/census } \\
\text { [accessed 22/03/2021] }\end{array}$ \\
\hline
\end{tabular}


Table 2. (Continued)

\begin{tabular}{|c|c|c|c|}
\hline Data source & Description & Data access & More information \\
\hline \multicolumn{4}{|l|}{ National surveys } \\
\hline $\begin{array}{l}\text { Office for National } \\
\text { Statistics (ONS) }\end{array}$ & $\begin{array}{l}\text { The ONS is an independent organisation } \\
\text { who collect, analyse, and disseminate sta- } \\
\text { tistics related to the economy, society and } \\
\text { population in the UK. In addition to the } \\
\text { census, data are collected through regular } \\
\text { and repeated individual and household } \\
\text { surveys, face-to-face interviews and tele- } \\
\text { phone interviews (such as the Annual } \\
\text { Population Survey and the Crime Survey } \\
\text { for England and Wales) which may be } \\
\text { combined with data from organisations } \\
\text { (such as the Department for Education } \\
\text { and policy recorded crime data). Surveys } \\
\text { are designed to be representative of peo- } \\
\text { ple or households living in England and } \\
\text { Wales. Includes individual-level annual } \\
\text { data of women and men aged 16-45 years } \\
\text { on wider determinants of health, emo- } \\
\text { tional and social health and support, } \\
\text { tobacco use and mental and physical } \\
\text { health. Data on alcohol use are collected } \\
\text { but not annually. }\end{array}$ & $\begin{array}{l}\text { Researchers can access the majority of } \\
\text { data through the UK Data Service, or data } \\
\text { may be available to accredited or } \\
\text { approved researchers for specific research } \\
\text { projects, and key statistics are published } \\
\text { online at least annually. }\end{array}$ & $\begin{array}{l}\text { https://www.ons.gov.uk/ } \\
\text { [accessed 22/03/2021] }\end{array}$ \\
\hline $\begin{array}{l}\text { British National Survey } \\
\text { of Sexual Attitudes and } \\
\text { Lifestyles (Natsal) }\end{array}$ & $\begin{array}{l}\text { Repeated cross-sectional surveys carried } \\
\text { out by a multi-disciplinary team of } \\
\text { researchers from University College } \\
\text { London, the London School of Hygiene \& } \\
\text { Tropical Medicine, the University of } \\
\text { Glasgow and NatCen Social Research. } \\
\text { Individual-level data are collected every } \\
10 \text { years from a random sample of } 10,000 \\
\text { adults aged } 16-74 \text { years living in private } \\
\text { households in the UK, and an additional } \\
\text { sample of } 5,000 \text { adults aged } 16-34 \text { years } \\
\text { for more detailed exploration of sexual } \\
\text { health and behaviours in this age-group. } \\
\text { Data are collected through face-to-face } \\
\text { interviews and self-completion question- } \\
\text { naires. Includes individual-level data of } \\
\text { women and men aged } 16-45 \text { years on } \\
\text { reproductive health and family planning, } \\
\text { health behaviours, immunisation and } \\
\text { infections, mental health conditions and } \\
\text { physical health conditions. }\end{array}$ & $\begin{array}{l}\text { Researchers can contact the Natsal study } \\
\text { team about collaboration on analysis of } \\
\text { Natsal data, which are available through } \\
\text { the UK Data Archive. Reference tables } \\
\text { with key statistics are published online for } \\
\text { each survey. }\end{array}$ & $\begin{array}{l}\text { https://www.natsal.ac.uk/ } \\
\text { [accessed 22/03/2021] }\end{array}$ \\
\hline $\begin{array}{l}\text { Health Survey for } \\
\text { England (HSE) }\end{array}$ & $\begin{array}{l}\text { Repeated cross-sectional surveys commis- } \\
\text { sioned by NHS Digital and carried out by } \\
\text { NatCen Social Research and University } \\
\text { College London to monitor the nation's } \\
\text { health and health-related behaviours. } \\
\text { Individual-level data are collected from a } \\
\text { random sample of adults aged } 16 \text { years } \\
\text { and over residing at private residential } \\
\text { addresses. Data are collected through } \\
\text { face-to-face interviews, self-completed } \\
\text { questionnaires and a follow-up nurse visit } \\
\text { to collect anthropometric measurements } \\
\text { and blood samples. Each year, approxi- } \\
\text { mately } 8,000 \text { adults complete the survey. } \\
\text { Includes individual-level annual data of } \\
\text { women and men aged } 16-45 \text { years on } \\
\text { wider determinants of health, health } \\
\text { behaviours, mental and physical health } \\
\text { conditions and medication. Data on con- } \\
\text { traception and physical activity are col- } \\
\text { lected but not annually. }\end{array}$ & $\begin{array}{l}\text { Researchers can access the majority of } \\
\text { data through the UK Data Service, and } \\
\text { key statistics are published online } \\
\text { annually. }\end{array}$ & $\begin{array}{l}\text { https://digital.nhs.uk/data-and- } \\
\text { information/publications/statistical/ } \\
\text { health-survey-for-england } \\
\text { [accessed 22/03/2021] }\end{array}$ \\
\hline $\begin{array}{l}\text { National Diet and } \\
\text { Nutrition Survey (NDNS) }\end{array}$ & $\begin{array}{l}\text { Repeated cross-sectional surveys carried } \\
\text { out on behalf of Public Health England } \\
\text { and the UK Food Standards Agency } \\
\text { collecting detailed individual-level infor- } \\
\text { mation on the food consumption, nutrient }\end{array}$ & $\begin{array}{l}\text { Researchers can access the majority of } \\
\text { data through the UK Data Service, and } \\
\text { results and time trends are published } \\
\text { online approximately every two years. }\end{array}$ & $\begin{array}{l}\text { https://www.gov.uk/government/ } \\
\text { collections/national-diet-and-nutrition- } \\
\text { survey } \\
\text { [accessed 22/03/2021] }\end{array}$ \\
\hline
\end{tabular}


Table 2. (Continued)

\begin{tabular}{|c|c|c|c|}
\hline \multirow[t]{2}{*}{ Data source } & Description & Data access & More information \\
\hline & $\begin{array}{l}\text { intake and nutritional status of the } \\
\text { general population living in private house- } \\
\text { holds in the UK. Data are collected } \\
\text { through an interview, a 4-day estimated } \\
\text { diet diary, physical measurements and a } \\
\text { blood and urine sample. Each year, data } \\
\text { are collected from } 500 \text { adults. Includes } \\
\text { individual-level annual data of women } \\
\text { and men aged } 16-45 \text { years on wider } \\
\text { determinants of health, nutrition and } \\
\text { weight. }\end{array}$ & & \\
\hline $\begin{array}{l}\text { Active Lives Adult } \\
\text { Survey (ALAS) }\end{array}$ & $\begin{array}{l}\text { Repeated cross-sectional surveys carried } \\
\text { out on behalf of government agencies } \\
\text { including Sport England, Arts Council } \\
\text { England and Public Health England by } \\
\text { Ipsos MORI collecting individual-level data } \\
\text { on how adults engage with sport and } \\
\text { physical activity. The survey is sent out to } \\
\text { a random sample of households across } \\
\text { England selected from the Royal Mail's } \\
\text { Postal Address File which has a very high } \\
\text { coverage of private residential addresses. } \\
\text { Each year, approximately } 175,000 \text { people } \\
\text { complete the survey. Data are weighted to } \\
\text { Office for National Statistics population } \\
\text { measures for geography and key demo- } \\
\text { graphics. Includes individual-level annual } \\
\text { data of women and men aged } 16-45 \text { years } \\
\text { on wider determinants of health, nutri- } \\
\text { tion, physical activity and weight. }\end{array}$ & $\begin{array}{l}\text { Researchers can access the majority of } \\
\text { data through the UK Data Service. Key } \\
\text { statistics are published online annually. }\end{array}$ & $\begin{array}{l}\text { https://www.sportengland.org/know- } \\
\text { your-audience/data/active-lives } \\
\text { [accessed 22/03/2021] }\end{array}$ \\
\hline \multicolumn{4}{|c|}{ National cohort studies } \\
\hline $\begin{array}{l}\text { Millennium Cohort } \\
\text { Study (MCS) }\end{array}$ & $\begin{array}{l}\text { Longitudinal population-based cohort } \\
\text { study following the lives of } 19,517 \text { children } \\
\text { born across England, Scotland, Wales and } \\
\text { Northern Ireland in } 2000-01 \text {. Since the ini- } \\
\text { tial birth survey, the cohort has been fol- } \\
\text { lowed up at ages } 3,5,7,11,14 \text { and } 17 \\
\text { years. Data are collected using question- } \\
\text { naires and interviewer-administered physi- } \\
\text { cal measurements. Includes individual- } \\
\text { level cross-sectional data (longitudinal } \\
\text { when additional follow-up surveys are } \\
\text { completed in the future) of women and } \\
\text { men aged } 17 \text { years on wider determinants } \\
\text { of health, emotional and social health and } \\
\text { support, reproductive health and family } \\
\text { planning, health behaviours, and physical } \\
\text { and mental health conditions. }\end{array}$ & $\begin{array}{l}\text { Researchers can access the majority of } \\
\text { data through the UK Data Service. }\end{array}$ & $\begin{array}{l}\text { https://cls.ucl.ac.uk/cls-studies/ } \\
\text { millennium-cohort-study/ } \\
\text { [accessed 22/03/2021] }\end{array}$ \\
\hline $\begin{array}{l}1970 \text { British Cohort } \\
\text { Study (BCS70) }\end{array}$ & $\begin{array}{l}\text { Longitudinal population-based cohort } \\
\text { study following the lives of more than } \\
17,000 \text { people born in England, Scotland } \\
\text { and Wales in a single week of } 1970 \text {. Since } \\
\text { the initial birth survey, the cohort has } \\
\text { been followed up at ages } 5,10,16,26,30 \text {, } \\
34,38,42 \text { and } 46 \text {. Data are collected using } \\
\text { questionnaires, interviews and assess- } \\
\text { ments. Includes individual-level data of } \\
\text { women and men aged } 16-42 \text { years on } \\
\text { wider determinants of health, emotional } \\
\text { and social health and support, reproduc- } \\
\text { tive health and family planning, health } \\
\text { behaviours and physical and mental } \\
\text { health conditions. }\end{array}$ & $\begin{array}{l}\text { Researchers can access the majority of } \\
\text { data through the UK Data Service. }\end{array}$ & $\begin{array}{l}\text { https://cls.ucl.ac.uk/cls-studies/1970- } \\
\text { british-cohort-study/ } \\
\text { [accessed 22/03/2021] }\end{array}$ \\
\hline
\end{tabular}

GP, general practitioner; NHS, National Health Service.

Data sources that were identified, but not considered relevant include the General Practice Quality Outcomes Framework (includes practice-level but not individual-patient-level data on

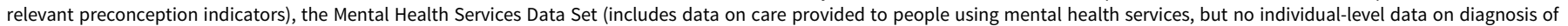
mental health conditions) and the Inflammatory Bowel Disease Registry (IBDR) (no individual-patient-level data available for research or accessible online). 
Perspective 1: Preconception care delivery in primary care

General practitioners (GPs) in England may provide preconception care (PCC) opportunistically, but they do not routinely provide PCC and no clinical PCC guidelines exist. GPs could play a leading role in the provision of PCC and make every contact count, but this requires a change in mindset. Routinely discussing pregnancy intentions, in particular during consultations for contraception, cervical screening and review of physical and mental health conditions and medication use such as for diabetes, asthma, epilepsy or depression, provides an opportunity to deliver PCC.

Data on prevalence and trends in preconception indicators at a national level may not be directly used by GPs when they care for patients with individual needs. However, surveillance data may contribute evidence to inform which indicators should be included in the Quality Outcomes Framework (QOF), and thereby influence the care GPs provide. Inclusion of one or two indicators, for example on body mass index and medication use among women and men planning pregnancy, may promote and normalise family planning conversations and awareness, and prompt an increase in the provision of PCC among GPs.

Dr Anne Connolly, general practitioner in Bradford, Chair of the Primary Care Women's Health Forum and RCGP Clinical Champion for Women's Health

Perspective 2: The role of health visitors in providing interconception care

Health visitors in England are registered nurses or midwives who work with families to give their children the best possible start to life by offering universal support and advice from the antenatal period until the child starts school at 5 years. While the focus of their work is on enhancing child health and development and reducing inequalities, the holistic approach of their service includes advice on parental factors that may affect child health. By addressing parental factors following childbirth such as recognising issues and advising on mental health, healthy family meals, housing and violence, health visitors contribute to provision of interconception care among couples who may have another pregnancy.

Monitoring of pre- and interconception health may identify factors that become more prevalent over time and that may therefore need additional attention and focus. For example, as a result of increases in obesity prevalence more resources may be developed to support healthy family meals and increases in postpartum mental health issues may prompt a more comprehensive assessment.

Sally Shillaker, Health Visitor, Academy of Research and Improvement, Solent NHS Trust

$$
\text { Perspective 3: Population-level preconception campaigns }
$$

The \#ReadyforPregnancy social media campaign was launched by the South East Clinical Delivery and Networks (SECDN) with a reach across South East England in June 2020. This 12-month campaign aims to support the goal of the National Maternity Transformation Programme (Better Births) to reduce stillbirth, neonatal and maternal deaths, by providing women and their partners with information on a specific preconception modifiable risk factor each month to encourage them to consider the importance of optimising these. Risk factors were based on preventive interventions reported in the National Maternity Transformation Programme, and messages were developed by the SECDN, Public Health England and NHS Creative, together with Local Maternity Systems and Maternity Voices Partnerships.

Information on prevalence and trends in preconception health indicators at a national level may be used to prioritise risk factor topics in a future campaign. Prevalence rates may also be incorporated in future campaign messages, for example to normalise sensitive topics such as mental health issues. East

measures based on evaluation of each measure against predetermined criteria (such as data quality and availability of comparable indicators across states), followed by a Delphi process among representatives from seven participating states to retain or exclude measures. ${ }^{32}$ The 45 measures have more recently also been evaluated and condensed to ten preconception health indicators ${ }^{33}$ and 30 preconception care indicators. ${ }^{34}$ This comprehensive consensus building process may explain why the measures identified by the PCHHC Initiative were more restricted compared to our overall set of identified indicators. For example, the PCHHC Initiative indicators were relevant to women only, did not include medication use, immunisation, genetic factors and environmental exposures and captured a limited number of measures to describe wider determinant of health and physical (chronic) health conditions. In line with our review, the PCHHC Initiative also identified preconception assessment and care (preconception counselling) as a relevant indicator. This is not currently reported in national data sources in England, but recorded in the Pregnancy Risk Assessment Monitoring System, which is a US state-based survey conducted between two and nine months postpartum among women with a live birth. ${ }^{32}$
There are some limitations and challenges to our review process and identified set of indicators. First, this is a scoping review and academic/scientific databases were not searched. We aimed to identify indicators based on grey literature including guidelines, recommendations, policy reports and other non-scientific documents from government organisations, national healthcare organisations, professional bodies and societies and charitable organisations. These documents were of interest as they are intended for the public or patients, healthcare professionals and governments, who may directly act on recommendations. These recommendations may thereby result in changes in preconception health that can be measured as part of surveillance. While the comprehensive list of indicators may not be exhaustive, it provides a strong foundation for the development of a preconception health surveillance system in England. Moreover, as evidence and awareness on the benefits of improved preconception health and care continues to grow, it is likely that indicators and measures will expand and be refined over time. Our identified set of indicators will therefore need regular review and updating. Furthermore, the aim of this review was to inform surveillance of indicators that can be assessed at the individual-level and indicators or measures 
at health service, practice, school, household or family-level were therefore beyond the scope of this review.

A number of indicators identified in our review are not relevant to all women of reproductive age, but are specific to women planning to become pregnant in the near future. Examples include taking a daily folic acid supplement, avoiding vitamin A (containing) supplements and adjusting medication that is not recommended in pregnancy. National routine data sources in England do not currently assess pregnancy intention, although there are moves to include such a measure in the national maternity services data set. $^{21}$ While the National Survey of Sexual Attitudes and Lifestyles (Natsal) includes a measure of pregnancy intention, this survey is only conducted every 10 years and includes a limited number of other preconception indicators that could be described and monitored among women with planned pregnancies. Until routine data sources include a measure of pregnancy intention, a comprehensive set of preconception indicators cannot be described and monitored separately in women intending to become pregnant. Although data on contraceptive use may provide an estimation of women 'at risk' of pregnancy, a better measure to assess pregnancy intention routinely is needed and research is underway to validate such a measure in the UK. ${ }^{35}$ Meanwhile, GPs or other primary care professionals can simply ask women “... and are you thinking of having a(nother) baby in the next year or so?", which can be incorporated as part of routine consultations to identify couples intending to become pregnant. ${ }^{36}$ Since the majority of indicators are relevant to the health and well-being of all people of reproductive age, optimising risk factors may improve pregnancy and birth outcomes if an intended or unintended pregnancy occurs, as well as a woman's health regardless of a future pregnancy.

In addition to the current lack of data on one indictor, national data sets may be subject to data quality issues. Incomplete data are a common problem, and depending on the amount and reason of missing data, this may result in biased prevalence estimates. Data quality is generally high in, for example, primary care and maternity data sets due to financial incentives to practices and service providers for achieving set targets. However, data from multiple sources may need to be combined to obtain more accurate estimates for some indicators, such as contraceptive use information from primary care, hospital and sexual and reproductive health services data sets. Assessment of indicators may change over time, for example, as a result of changes in quality indicators for primary care, data structures and content requirements for health services, or survey questions. This may impact the accuracy of monitoring of changes in preconception health and care over time.

While acknowledging these potential limitations, surveillance of preconception indicators can be used to provide a snapshot of the health and health behaviours of women and men of reproductive age. When these indicators are stratified by wider determinants of health, they provide an understanding of needs and disparities in health and health care among subpopulations. Researchers may use the overview of indicators to identify outcomes to be targeted and assessed in intervention studies that aim to improve preconception health. Moreover, routine reporting on population-level indicators that benchmark the health of reproductive-aged people can be used to enhance accountability from governments and other relevant agencies for delivering interventions that remove barriers and support all women and men to improve their health. ${ }^{21}$ The importance and utility of preconception health surveillance is further highlighted by the COVID-19 pandemic, when the prevalence of many indicators identified in this review is likely affected as a result of the stay-at-home advice and changes in access to health care services. ${ }^{37}$ Investment in efficient routine data collection and monitoring of population, maternal and child health is recommended to determine the immediate and longer-term effects of COVID-19 and the additional needs of people of reproductive age. ${ }^{38,39}$

The implications of preconception surveillance extend beyond research and policy advocacy, to directly and indirectly improve patient care and inform the development and evaluation of education and awareness campaigns. Discussions with relevant stakeholders (Fig. 1) revealed that, for example, in primary care, surveillance data may indirectly influence patient care through the Quality Outcomes Framework (QOF). ${ }^{40}$ This is a voluntary annual reward and financial incentive programme for general practitioner (GP) services in England, in which practices can score points based on achievements against a set of indicators. The QOF does not currently include preconception indicators, but high prevalence or increasing trends may inform the inclusion of specific indicators, and thereby contribute to improved provision of preconception care among GPs. ${ }^{41}$ Moreover, health visitors in England contribute to the provision of interconception care to mothers with infants and young children and their partners. Prevalence and trend data on pre- and interconception indicators may inform risk assessments performed by health visitors and indicate the need to develop resources to address issues that are (increasingly) common and may affect the next pregnancy. In addition to patient care, surveillance of preconception indicators may be used to inform campaign development and evaluation by identifying common indicators that could be targeted and would be relevant to a large proportion of the population. Prevalence rates of indicators may also be communicated to the target population for education purposes and to normalise sensitive topics such as mental health issues.

The identification of a comprehensive list of preconception indicators and determining their availability in national data sources are a first step towards national surveillance of preconception health in England. Analyses of routine health data sets are currently underway to obtain national prevalence estimates of preconception indicators. Further planned work includes linkage of these data sets to allow analyses of associations between preconception indicators and maternal and child health outcomes to provide evidence on the potential impact of improved preconception health. Our findings on the prevalence of indicators and strength of associations with relevant outcomes - together with considerations of data quality, modifiability and input from stakeholder and public consultations - will inform the prioritisation of a reduced set of key indicators for ongoing surveillance of preconception health in England. A dashboard bringing indicators from multiple sources together through an interactive online platform will make findings readily available to health care providers, policy makers, the public and relevant stakeholders. Informed by the list of preconception indicators presented in this review and by the PCHHC Initiative, we recommend other countries follow a similar process to develop a surveillance system for preconception health based on available regional or national data sets.

\section{Conclusion}

Several national and international guideline and policy documents emphasise the need to optimise preconception health to prevent adverse outcomes in the next generation and improve the overall health and well-being of women and men of reproductive age 
irrespective of any pregnancies they may have. Nearly all preconception indicators identified from these documents can be described and monitored using national data sources in England. Measures of preconception health assessment and care and pregnancy intention are not currently annually recorded in any national data source and should be considered for inclusion in routine data sets or annual surveys in the future. Monitoring of a comprehensive set of preconception indicators may contribute to informing patient care, developing and evaluating public awareness campaigns and strengthening advocacy efforts for government resources and action. This work informs the next steps towards developing a national surveillance system and to improve preconception health in England.

Supplementary material. To view supplementary material for this article, please visit https://doi.org/10.1017/S2040174421000258

Acknowledgements. The UK Preconception Partnership is a coalition of groups representing different aspects of preconception health in women and their partners, including the Royal College of General Practitioners, the Royal College of Obstetricians and Gynaecologists, the Faculty of Sexual \& Reproductive Healthcare, Public Health England, Tommy's charity and academics in reproductive and sexual health, obstetrics and gynaecology, population health and epidemiology, nutritional sciences and behavioural sciences and education in schools.

Financial support. DS is supported by the NIHR Southampton Biomedical Research Centre (IS-BRC-1215-20004). KG is supported by the UK Medical Research Council (MC_UU_12011/4), the National Institute for Health Research (NIHR Senior Investigator (NF-SI-0515-10042), NIHR Southampton 1000DaysPlus Global Nutrition Research Group (17/63/154), NIHR Southampton Biomedical Research Centre (IS-BRC-1215-20004), the European Union (Erasmus+ Programme ImpENSA) and the British Heart Foundation (RG/15/17/3174).

Conflicts of interest. KG has received reimbursement for speaking at conferences sponsored by companies selling nutritional products, and is part of an academic consortium that has received research funding from Abbott Nutrition, Nestec, BenevolentAI Bio Ltd. and Danone. The other authors have no conflicts of interest to disclose.

\section{References}

1. Fleming TP, Watkins AJ, Velazquez MA, et al. Origins of lifetime health around the time of conception: causes and consequences. Lancet. 2018; $391,1842-1852$.

2. Stephenson J, Heslehurst N, Hall J, et al. Before the beginning: nutrition and lifestyle in the preconception period and its importance for future health. Lancet. 2018; 391, 1830-1841.

3. National Institute for Health and Care Excellence (NICE). Pre-conception - advice and management. 2019. Available from: https://cks.nice.org.uk/ pre-conception-advice-and-management. [accessed 22/03/2021].

4. National Health Service (NHS). Planning your pregnancy. 2020. Available from: https://www.nhs.uk/conditions/pregnancy-and-baby/planningpregnancy/. [page last reviewed 21/01/2020] [accessed 22/03/2021].

5. National Institute for Health Research (NIHR). Themed Review: Better Beginnings. 2017. Available from: https://evidence.nihr.ac.uk/wpcontent/uploads/2020/03/Better-beginnings-web-interactive.pdf. [accessed 22/03/2021].

6. Centers for Disease Control and Prevention (CDC). Recommendations to Improve Preconception Health and Health Care - United States. A Report of the CDC/ATSDR Preconception Care Work Group and the Select Panel on Preconception Care. 2006. Available from: https://www.cdc.gov/mmwr/ preview/mmwrhtml/rr5506a1.htm. [accessed 22/03/2021].

7. Public Health Agency of Canada. Family-Centred Maternity and Newborn Care: National Guidelines. Chapter 2. Preconception care. 2019. Available from: https://www.canada.ca/en/public-health/services/publications/healthy- living/maternity-newborn-care-guidelines-chapter-2.html. [accessed 22/03/ 2021].

8. Jack BW, Atrash H, Coonrod DV, Moos MK, O'Donnell J, Johnson K. The clinical content of preconception care: an overview and preparation of this supplement. Am J Obstet Gynecol. 2008; 199(6 Suppl 2), S266-S279.

9. World Health Organization (WHO). Preconception care. Maximizing the gains for maternal and child health. Policy Brief. 2012. Available from: https://www.who.int/maternal_child_adolescent/documents/preconception_ care_policy_brief.pdf. [accessed 22/03/2021].

10. Public Health England. Making the case for preconception care. 2018. Available from: https://assets.publishing.service.gov.uk/government/ uploads/system/uploads/attachment_data/file/729018/Making_the_case_ for_preconception_care.pdf [accessed 22/03/2021].

11. Royal Australian and New Zealand College of Obstetricians and Gynaecologists (RANZCOG). Pre-pregnancy counselling - statement. 2017. Available from: https://ranzcog.edu.au/RANZCOG_SITE/media/ RANZCOG-MEDIA/Women\%27s\%20Health/Statement\%20and\% 20guidelines/Clinical-Obstetrics/Pre-pregnancy-Counselling-(C-Obs3a)-review-July-2017_1.pdf?ext=.pdf. [accessed 22/03/2021].

12. Faculty of Sexual and Reproductive Healthcare (FSRH). Statement from the Clinical Effectiveness Unit. Pre-conception Care. 2016. Available from: https://www.fsrh.org/standards-and-guidance/documents/pre-con ception-care-care-ceu-statement-august-2016/. [accessed 22/03/2021].

13. American College of Obstetricians and Gynecologists (ACOG). Clinical guidance. Committee opinion of preconception counceling. 2019. Available from: https://www.acog.org/clinical/clinical-guidance/committeeopinion/articles/2019/01/prepregnancy-counseling. [accessed 22/03/2021].

14. United Nations. Transforming our World: The 2030 Agenda for Sustainable Development, 2015. New York: United Nations. Available from https:// sustainabledevelopment.un.org/post2015/transformingourworld/ publication [accessed 22/03/2021].

15. World Health Organization (WHO). Global Strategy for Women's, Children's and Adolescent's Health, 2016-2030, 2015. New York: United Nations. Available from https://www.who.int/life-course/partners/globalstrategy/en/. [accessed 22/03/2021].

16. Jacob CM, Newell M-L, Hanson M. Narrative review of reviews of preconception interventions to prevent an increased risk of obesity and non-communicable diseases in children. Obes Rev. 2019; 20(S1), 5-17. doi: 10.1111/ obr.12769

17. Public Health England. Health of women before and during pregnancy: health behaviours, risk factors and inequalities. 2019. Available from: https://assets.publishing.service.gov.uk/government/uploads/system/ uploads/attachment_data/file/844210/Health_of_women_before_and_ during_pregnancy_2019.pdf. [accessed 22/03/2021].

18. Ebrahim SH, Lo SS-T, Zhuo J, Han J-Y, Delvoye P, Zhu L. Models of preconception care implementation in selected countries. Matern Child Health J. 2006; 10(5 Suppl), S37-S42.

19. Wald NJ, Morris JK, Blakemore C. Public health failure in the prevention of neural tube defects: time to abandon the tolerable upper intake level of folate. Public Health Rev. 2018; 39, 1-11.

20. Walani SR, Moley KH. Global strategies for change. In Preconception Health and Care: A Life Course Approach (eds. Shawe J, Steegers E, Verbiest S), 2020; pp. 287-297. Springer, Cham.

21. Stephenson J, Vogel C, Hall J, et al. Preconception health in England: a proposal for annual reporting with core metrics. Lancet. 2019; 393, 2262-2271.

22. National Health Service (NHS) Digital. Data collections and data sets. Available from https://digital.nhs.uk/data-and-information/data-collectionsand-data-sets. [accessed 22/03/2021].

23. UK Data Service. Available from https://ukdataservice.ac.uk/. [accessed 22/ 03/2021].

24. Public Health England. Public Health Outcomes Framework. Available from: https://fingertips.phe.org.uk/profile/public-health-outcomes-framework. [accessed 22/03/2021].

25. Office for National Statistics (ONS). Available from: https://www.ons.gov. $\mathrm{uk} /$. [accessed 22/03/2021] 
26. Federation of Obstetric and Gynaecological Societies of India (FOGSI). Good Clinical Practice Recommendation on Preconception Care. 2015. Available from: https://www.fogsi.org/gcpr-preconception-care/. [accessed 22/03/2021]

27. Ontario Public Health Association. Enhancing the Health of Ontarians: A Call to Action for Preconception Health Promotion \& Care, 2014. Toronto, ON. Available from: https://opha.on.ca/getmedia/2da52762-6614-40f797ff-f2a5043dea21/OPHA-Shift-Enhancing-the-health-of-Ontarians-Acall-to-action-for-preconception-health-promotion-and-care_1.pdf.aspx? ext $=$.pdf [accessed 22/03/2021].

28. National Health Service (NHS) Greater Glasgow and Clyde. Prepared for pregnancy? Preconception health,education and care in Scotland. 2016. Available from: https://www.stor.scot.nhs.uk/handle/11289/578820. [accessed 22/03/2021].

29. Centers for Disease Control and Prevention (CDC). Before pregnancy. 2020. Available from: https://www.cdc.gov/preconception/index.html. [page last reviewed February 2020] [accessed 22/03/2021].

30. National Institute for Health and Care Excellence (NICE). Fertility problems: assessment and treatment. Clinical guideline [CG156]. 2013. Available from: https://www.nice.org.uk/guidance/cg156. [last updated 06/09/2017] [accessed 22/03/2021].

31. Tommy's. Planning a pregnancy. 2017. Available from: https://www. tommys.org/pregnancy-information/planning-pregnancy [accessed 22/ 03/2021].

32. Broussard DL, Sappenfield WB, Fussman C, Kroelinger CD, Grigorescu V. Core state preconception health indicators: a voluntary, multi-state selection process. Matern Child Health J. 2011; 15, 158-168.

33. Robbins CL, D'Angelo D, Zapata L, et al. Preconception Health Indicators for Public Health Surveillance. J Womens Health. 2018; 27, 430-443.

34. The Surveillance and Research Workgroup and Clinical Workgroup of the National Preconception Health and Health Care Initiative. Surveillance indicators for women's preconception care. J Womens Health. 2020; 29, 910-918.

35. Rocca CH, Ralph LJ, Wilson M, Gould H, Foster DG. Psychometric evaluation of an instrument to measure prospective pregnancy preferences: the desire to avoid pregnancy scale. Med Care. 2019; 57, 152-158.

36. Stephenson J, Schoenaker D, Hinton W, et al. A wake-up call for preconception health: a clinical review. Br J Gen Pract. 2021; 71(706), 233-236.

37. Public Health England. Wider Impacts of COVID-19 on Health (WICH): monitoring tool. Available from: https://fingertips.phe.org.uk/profile/ covid19. [accessed 22/03/2021].

38. Jacob CM, Briana DD, Di Renzo GC, et al. Building resilient societies after COVID-19: the case for investing in maternal, neonatal, and child health. Lancet Public Health. 2020; 5, e624-e627.

39. Roseboom TJ, Ozanne SE, Godfrey KM, et al. Unheard, unseen and unprotected: $\mathrm{DOHaD}$ council's call for action to protect the younger generation from the long-term effects of COVID-19. J Dev Orig Health Dis. 2021; 12, 3-5.

40. National Health Service (NHS) Digital. Quality Outcomes Framework (QOF). Available from: https://digital.nhs.uk/data-and-information/data- tools-and-services/data-services/general-practice-data-hub/qualityoutcomes-framework-qof. [page last edited 21/08/2020] [accessed 22/ 03/2021].

41. Ma R, Cecil E, Bottle A, French R, Saxena S. Impact of a pay-for-performance scheme for long-acting reversible contraceptive (LARC) advice on contraceptive uptake and abortion in British primary care: an interrupted time series study. PLoS Med. 2020; 17, e1003333.

42. National Institute for Health and Care Excellence (NICE). Diabetes in pregnancy: management from preconception to the postnatal period. NICE guideline [NG3]. 2015. Available from: https://www.nice.org.uk/ guidance/ng3. [last updated 26/08/2015] [accessed 22/03/2021].

43. National Institute for Health and Care Excellence (NICE). Hypertension in pregnancy. Quality standard [QS35]. 2013. Available from: https://www. nice.org.uk/guidance/qs35. [last updated 23/07/2019] [accessed 22/03/ 2021].

44. National Health Service (NHS) Health Education England. E-learning programme on Healthy Pregnancy Pathways: Pre-conception care. Available from: https://www.e-lfh.org.uk/heathy-pregnancy-pathway/preconception/index.html. [accessed 22/03/2021].

45. National Institute for Health and Care Excellence (NICE). Eating disorders. 2019. Available from: https://cks.nice.org.uk/eating-disorders\#!scenario:1. [last revised July 2019] [accessed 22/03/2021].

46. Royal College of Obstetricians and Gynaecologists (RCOG). Cardiac disease and pregnancy. Good practice no. 13. 2011. Available from: https://www. rcog.org.uk/globalassets/documents/guidelines/goodpractice13cardiac diseaseandpregnancy.pdf. [accessed 22/03/2021].

47. Gardiner PM, Nelson L, Shellhaas CS, et al. The clinical content of preconception care: nutrition and dietary supplements. Am J Obstet Gynecol. 2008; 199(6 Suppl 2), S345-S356.

48. National Institute for Health and Care Excellence (NICE). Weight management before, during and after pregnancy. Public health guideline [PH27]. 2010. Available from: https://www.nice.org.uk/guidance/ph27. [accessed 22/03/2021].

49. Frey KA, Navarro SM, Kotelchuck M, Lu MC. The clinical content of preconception care: preconception care for men. Am J Obstet Gynecol. 2008; 199(6 Suppl 2), S389-S395.

50. National Institute for Health and Care Excellence (NICE). Endometriosis: diagnosis and management. NICE guideline [NG73]. 2017. Available from: https://www.nice.org.uk/guidance/ng73. [accessed 22/03/2021].

51. Faculty of Sexual and Reproductive Healthcare (FSRH). Clinical Guideline: SRH for Individuals with Inflammatory Bowel Disease (IBD). 2016 Available from: https://www.fsrh.org/standards-and-guidance/docu ments/ceu-clinical-guidance-srh-ibd/. [accessed 22/03/2021].

52. Atrash H, Jack B. Preconception care to improve pregnancy outcomes: clinical practice guidelines. J Hum Growth Dev. 2020; 30, 407-416.

53. de Lusignan S, Correa A, Smith GE, et al. RCGP Research and Surveillance Centre: 50 years' surveillance of influenza, infections, and respiratory conditions. Br J Gen Pract. 2017; 67, 440-441.

54. Herrett E, Gallagher AM, Bhaskaran K, et al. Data Resource Profile: Clinical Practice Research Datalink (CPRD). Int J Epidemiol. 2015; 44, 827-836. 